\title{
Control sharing in human-robot team interaction
}

\author{
Selma Musić ${ }^{1}$, Sandra Hirche ${ }^{2}$ \\ Technische Universität München, Munich, Germany
}

\begin{abstract}
Human-robot interaction is a wide area of research which exploits complementary competences of humans and robots. Humans are capable of reasoning and planning, while robots are capable of performing tasks repetitively and precisely. Rapid developments in robotics and reduction of cost lead to an increased research interest in the area of robot teams and human-robot team interaction. One of the key research questions is how to combine human and robot team decision making and task execution capabilities, i.e. how control should be shared among them. This paper surveys advances in human-robot team interaction with special attention devoted to control sharing methodologies. Additionally, aspects affecting the control sharing design, such as robot team and human behavior modeling, level of autonomy and human-machine interfaces are identified. Open problems and future research directions towards joint decision making and task execution in human-robot teams are discussed.
\end{abstract}

Keywords: Human-robot team interaction, shared control, human behavior modeling, robot team

\section{Introduction}

Human-robot team interaction describes the interaction between a human and multiple robots, which collaborate to achieve a common goal. Its envisioned benefits are superior performance in highly unstructured tasks in unknown and/or remote environments, reduced human workload, execution of tasks which are not possible with a single robot, flexibility in task execution, and robustness. Application domains of human-robot team interaction include for example: search and rescue [57], cooperative manipulation [63], collaborative manufacturing, logistics, and construction.

Rapid technological developments in the area of autonomous robotics result in large improvements of robots' reliability and adaptability to unknown environments. With these developments the nature of human-robot interaction changes, as robots become smart tools to humans, or even their collaborative partners.

Reduction of price, size, and operational complexity considerably increases the availability of modern robots, while the advancements in communica-

\footnotetext{
${ }^{1}$ selma.music@tum.de

2hirche@tum.de

tion technology allow a seamless information exchange between them. These developments are enablers for multi-robot systems. They provide increased flexibility and robustness and are capable to conduct more complex tasks then single-robot systems [90].

Even though the capabilities of modern robots are enhanced, they still need human intervention in the form of high-level reasoning and planning. As a consequence, novel forms of human-robot interaction beyond single-human-single-robot have become a current and important topic of research: multiple humans-single robot interaction [73], multiple humans-multiple robots interaction [43], and single human-multiple robots interaction [26].

The main scientific challenge of human-robot team interaction is to fuse the cognitive capabilities of the human and the autonomous capabilities of the robot team, while maximizing task performance and intuitiveness of the interaction. This leads to the consideration of suitable levels of autonomy, control sharing and human cognitive and behavioral aspects in the interaction design.

The aim of this article is to provide a survey on the existing literature on human-robot team interaction with the special focus on its control sharing aspects. The overview of the article structure is

July 15, 2017 


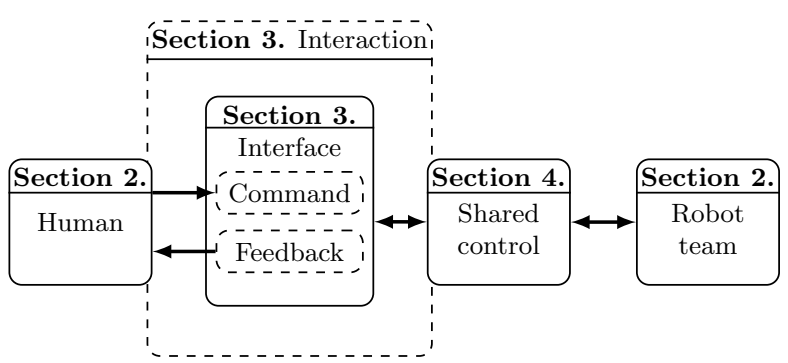

Figure 1: Article overview in a block structure.

provided with the block scheme in Figure 1. We review relevant literature considering important research challenges for each component of the shared control loop for human-robot team interaction.

\section{Robot team and human aspects in the in- teraction}

In this section we briefly review modeling and control approaches for robot teams and human behavior modeling.

\subsection{Modeling and control of robot teams}

This subsection focuses on the modeling and control concepts for robot teams, which are suitable and/or used in human-robot team interaction. Extensive reviews on multi-robot systems in general are, among many [33], [39], [47] and [79].

In this article the term robot team refers to a multirobot system which cooperates to perform a global task. Robot teams can be a set of mobile manipulators [108], wheeled robots [27], UAVs [56]. A swarm is considered as a type of robot team which contains relatively large number of "simple" and homogeneous robots. There are also heterogeneous cooperative multi-robot systems, which we here include under the term robot teams as well.

Depending on the coupling between the individual robots, robot teams can be uncoupled, loosely coupled or tightly coupled (e.g. through physical constraints) systems. Uncoupled and loosely coupled robot teams are modeled as a set of differential equations describing the models of individual robots. Most frequently used models are:

- Kinematic (single integrator) model [111]:

$$
\dot{\boldsymbol{x}}_{i}=\boldsymbol{u}_{i} \quad i=1, \ldots, N,
$$

where $\boldsymbol{x}_{i} \in \mathbb{R}^{n}$ is the pose of the $i$-th robot, $\boldsymbol{u}_{i} \in$ $\mathbb{R}^{n}$ its control input, and $N$ the number of robots.
- Point mass (double integrator) model [71]:

$$
\ddot{\boldsymbol{x}}_{i}=\frac{1}{m_{i}} \boldsymbol{u}_{i},
$$

where $m_{i}$ is the mass of the $i^{\text {th }}$ robot.

- Euler-Lagrange model [81]:

$$
M_{i}\left(\boldsymbol{q}_{i}\right) \ddot{\boldsymbol{q}}_{i}+\boldsymbol{c}\left(\boldsymbol{q}_{i}, \dot{\boldsymbol{q}}_{i}\right)+\boldsymbol{g}_{i}\left(\boldsymbol{q}_{i}\right)=\boldsymbol{\tau}_{i},
$$

where $\boldsymbol{q}_{i} \in \mathbb{R}^{n}$ is the vector of generalized coordinates, $\boldsymbol{M}_{i}\left(\boldsymbol{q}_{i}\right) \in \mathbb{R}^{n \times n}$ is the inertia matrix, $\boldsymbol{c}\left(\boldsymbol{x}_{i}, \dot{\boldsymbol{x}}_{i}\right) \in \mathbb{R}^{n}$ the vector of Coriolis and centrifugal forces, $\boldsymbol{g}_{i}\left(\boldsymbol{x}_{i}\right) \in \mathbb{R}^{n}$ the vector of gravitational forces, and $\boldsymbol{\tau}_{i} \in \mathbb{R}^{n}$ is the vector of control torques.

- State-space model:

$$
\dot{\boldsymbol{x}}_{i}=\boldsymbol{f}_{i}\left(\boldsymbol{x}_{i}, \boldsymbol{u}_{i}\right)
$$

where $\boldsymbol{f}_{i} \in \mathbb{R}^{n}$ is a smooth vector field representing the dynamics of the robot.

For tightly coupled robot teams it is necessary to model the physical interactions between the individual robots, for example see [37]. Together with continuous states, a discrete state, termed as role [79], can be assigned to each robot in the team. The role can refer to a set of responsabilities or capabilities a robot has within the team [116], and is particularly relevant for heterogeneous teams. Roles can also determine to what extent the individual robots are capable of making decisions. Examples are leader and follower roles, where a leader does not use information of other robots to make a decision, while a follower considers the information of other robots to make its decision.

\subsubsection{Control of robot teams}

Control architectures for robot teams largely depend on the way in which robots interact to achieve team behaviors. In this context, it is possible to distinguish between centralized and distributed control approaches. Centralized control architectures command the team from a single point (e.g. through the robot leader). Therefore, they have a single point of failure. Distributed control architectures run locally on the robots and communicate between themselves. Achieving team behaviors is more challenging in this case, but the reliability is higher. For human-robot team interaction, a combination of centralized (through the human involvement) and 


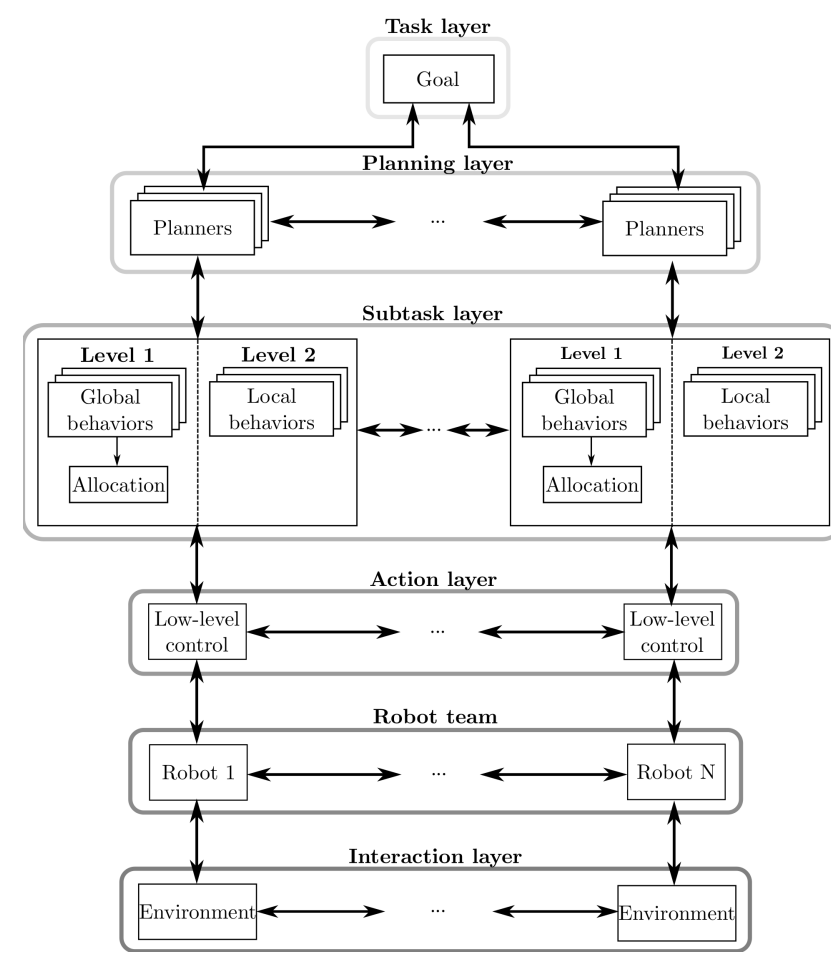

Figure 2: Hierarchical control architecture for robot teams. Goal of the robot team is determined and monitored in the task layer. Based on the goal, a set of global and local behaviors are activated in the subtask layer. The outputs of this layer are control inputs for the low-level controllers of robots in the action layer.

distributed (between the robots) control is the most suitable.

An illustration of a control architecture for robot teams, which can be extended to the human-robot team interaction, is depicted with Figure 2. It is possible to distinguish between 6 layers within the architecture: task, planning, subtask, action, robot teams and interaction layers. The planning layer is not treated in this article.

The knowledge about the goal (task, mission) is stored within the task layer. Often the task is defined as a performance function [79]:

$$
J=\int_{0}^{T} L(\boldsymbol{x}, \boldsymbol{\alpha}, \boldsymbol{u}) d t+V(\boldsymbol{x}(T), \boldsymbol{\alpha}(T))
$$

where $L$ and $V$ are incremental and terminal costs, respectively. Continuous states of the robots, $\boldsymbol{x}_{i}$, are stacked in the vector $\boldsymbol{x} \in \mathbb{R}^{n N}$, discrete states (roles) are denoted by $\boldsymbol{\alpha}$, the control inputs are $\boldsymbol{u}=\boldsymbol{\gamma}(\boldsymbol{x}, \boldsymbol{\alpha})$ with $\boldsymbol{\gamma}$ being a smooth vector field, while $T$ is the time horizon in which the task should be accomplished.
The elementary behaviors or subtasks are stored within the subtask layer. We consider that global behaviors require information exchange between the robots, and local require only the local information of a robot. Some of the global behaviors are rendezvous, aggregation, foraging, cooperative manipulation, formation, coverage, inter-robot avoidance, etc. An important local behavior is $o b$ stacle avoidance.

Rendezvous describes a behavior in which the robots meet at a common point at a common time [79]. Foraging refers to a behavior of collecting and delivering an object. Formation refers to the maintenance of robot poses relative to each other or to a reference [34], [35], [66]. Coverage refers to the use of the team to cover and visit areas of an environment for information acquisiton [23]. Combinations of these, elementary behaviors, can define different missions. For example, flocking incorporates aggregation and avoidance as a set of necessary behaviors [84].

Global behaviors are achieved through cooperation. Coordination control approaches from the area of multi-agent systems are suitable for accomplishing global behaviors by exchanging individual state information through the network of agents (robots) to reach a common agreement/consensus [85]. For example, in order to accomplish a rendezvous behavior, the robots need to perform consensus on the position. The idea behind the consensus control is that each robot moves towards the weighted average of the states of its neighbors. Communication topology of the robot team is frequently modeled with graphs [40]. Robustness towards topology changes and communication uncertainties such as packet loss and time delay are important control challenges. There are multiple other control approaches that are used for cooperation of robot teams, e.g. artifical potential functions [66], Lyapunov analysis [85], sliding mode control [46], behavioral control [4], virtual structures [67], to name the few.

Nowadays, robot teams need to operate in dynamic, unstructured environments. Therefore, for a successfull execution of the task, multiple subtasks need to be performed simultaneously. In order to achieve this, a behavior-based control approach is suitable [7]. It is designed by defining and weighting the elementary behaviors. Subtasks are defined 
as transformations of the system states

$$
\begin{aligned}
& \boldsymbol{x}_{t_{i}}=\boldsymbol{f}_{t_{i}}(\boldsymbol{x}) \\
& \dot{\boldsymbol{x}}_{t_{i}}=\boldsymbol{J}_{t_{i}}(\boldsymbol{x}) \dot{\boldsymbol{x}}
\end{aligned}
$$

where $\boldsymbol{x}_{t_{i}}$ are the coordinates of the subtask $t_{i}$ and $\boldsymbol{J}_{t_{i}}(\boldsymbol{x})$ is the corresponding subtask Jacobian. Therefore, behavioral control defines global and local behaviors as subtask functions.

Example 2.1. In order to manipulate a common object in $\mathbb{R}^{2}$ from an initial to a final configuration, a team of robot manipulators needs to collectively move to a desired location, while maintaining a fixed formation. Therefore, we can define two subtask functions

$$
\begin{aligned}
\boldsymbol{e}_{m}(\boldsymbol{x}) & =\frac{1}{N} \Sigma_{i=1}^{N} \boldsymbol{x}_{i}-\boldsymbol{x}_{m}^{d}, \\
\boldsymbol{e}_{f}(\boldsymbol{x})= & {\left[\begin{array}{c}
\left(\boldsymbol{x}_{2}-\boldsymbol{x}_{1}\right)^{T}-\boldsymbol{d}_{12}^{d} \\
\vdots \\
\left(\boldsymbol{x}_{N}-\boldsymbol{x}_{N-1}\right)-\boldsymbol{d}_{(N-1) N}^{d}
\end{array}\right], }
\end{aligned}
$$

where $\boldsymbol{x}_{m}^{d}$ is the desired mean position of the robot team and $\boldsymbol{d}_{(i-1) i}^{d}$ is the desired distance between robots $i-1$ and $i$.

A behavioral control approach that ensures subtasks are conducted according to a predefined priority is termed as Null-space based behavioral control [4]. A common approach is to project lower priority subtasks onto the null-space of the higher priority subtask. For example, in the case of 2 subtasks, the control input $\dot{\boldsymbol{x}}^{d}$ would be:

$$
\dot{\boldsymbol{x}}^{d}=\boldsymbol{J}_{t_{1}}^{\dagger} \boldsymbol{x}_{t_{1}}+\left(\boldsymbol{I}-\boldsymbol{J}_{t_{1}}^{\dagger} \boldsymbol{J}_{t_{1}}\right) \boldsymbol{J}_{t_{2}}^{\dagger} \boldsymbol{x}_{t_{2}},
$$

where $\left(\boldsymbol{I}-\boldsymbol{J}_{t_{1}}^{\dagger} \boldsymbol{J}_{t_{1}}\right)$ is the null-space projector. However, the approach is kinematic, which makes it unsuitable for the control of dynamic behaviors (e.g. when the inertia of the team cannot be neglected). Additionally, interaction with the environment (e.g. with objects or humans) cannot be handled appropriately.

Allocation of responsibilities to the individual robots, according to the selected subtasks, and the role they have within the team is an important step and can be handled in various ways, see for example [49] and [117].

In order to exploit human capabilities within the control architecture for robot teams, it is necessary to assign a role to the human, understand behaviors and constraints of the human in the interaction, and

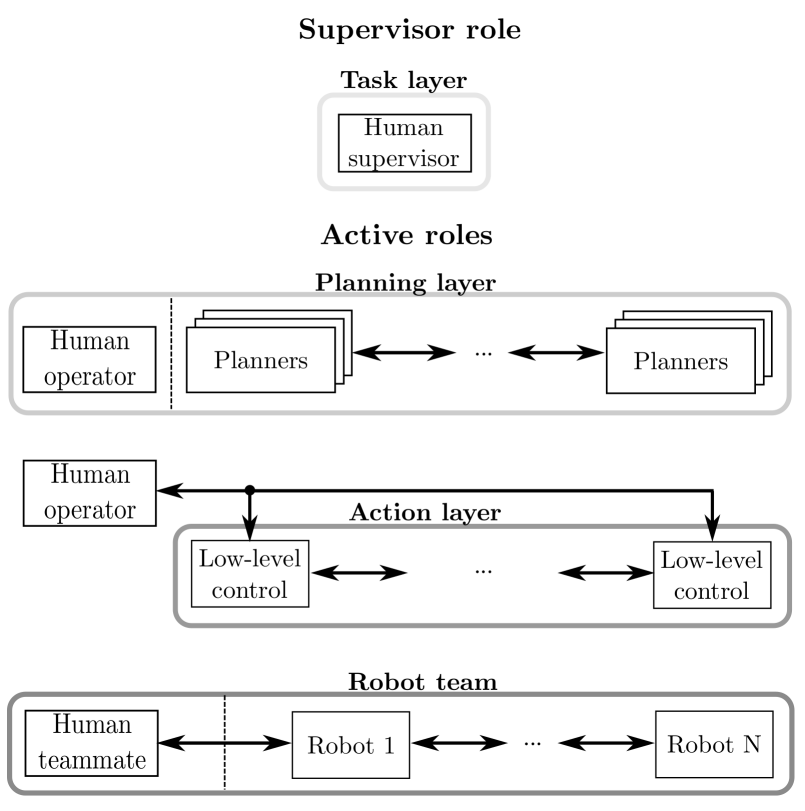

Figure 3: Possible human roles within the robot team control architecture.

how the control approaches for robot teams need to be extended towards human-robot team interaction.

\subsection{Human behavior modeling}

The human is an element of the control loop in human-robot team interaction, see Figure 5. Based on the team states, delivered through a feedback interface, the human performs an action, mapped into a command for the robot team through the command interface.

In order to establish the human-robot team interaction, the concepts introduced in Subsection 2.1 need to be extended to the human by assigning him/her an appropriate role. We distinguish between supervisory and active human role [18], depicted in Figure 3.

A supervisory role brings human on the loop and considers the interaction on a symbolic and discrete level. The human supervisor is located on the task layer. Therefore, the human is aware of the overall goal and is capable of modifying it. The responsibilities of the supervisor are to select global and local behaviors and intervene when necessary.

An active role brings human in the control loop with the robot team and considers their interaction on a physical and continuous level [64]. Active role can be exhibited in the form of the human operator which provides control inputs to the subtask or 
action layers, or it can be exhibited by including human in the team.

Very frequently the human behavior in the interaction is not modeled. Instead, assumptions are imposed on the human expertise in both roles and on human passivity in the active role, e.g. [104]. An appropriate human model can predict under which conditions the human exhibits bad performance or instability, and may be beneficial in the design of appropriate control sharing. With the increase of robot team capabilities, human cognitive models are needed. Relevant models and constraints on the human behavior in the interaction are found within the area of cognitive psychology. These models can provide valuable insight, but need to be transformed into the form suitable for systems and control analysis.

Very often the human supervisor is modeled by a Markov model. In [110], a Markov model is obtained from the neurally inspired cognitive model. It predicts the human decision when choosing between two global behaviors of the swarm, Deploy (D) or Rendezvous (R). The probability of transition from one behavior to another is proposed as

$$
p_{s_{i} \rightarrow s_{j}}=\frac{c_{s_{i} \rightarrow s_{j}}}{\Sigma_{s_{t} \in\{D, R\}} c_{s_{i} \rightarrow s_{j}}}
$$

where $s_{i}, s_{j} \in\{D, R\}$ are two possibilities of the team behavior, and $c_{s_{i} \rightarrow s_{j}}$ is the number of transitions from $s_{i}$ to $s_{j}$ obtained during the training of the Markov model. The prediction of the next chosen behavior is obtained with

$$
s_{i+1}=\underset{x \in\{D, R\}}{\arg \max } p_{s_{i} \rightarrow x} .
$$

Models that can capture the dynamics of the decision making are termed as accumulator models [91]. They are suitable for modeling the human decision-making behavior in the supervisory as well as the active role. The accumulator models are typically used for two-alternative forced-choice tasks (TAFCTs).

The authors of [45] formulate an extended decision field theory (EDFT) model to represent multiple sequential decisions in human-automation interaction with supervisory role. The preference in two-forced choice tasks at sample $n$ is proposed as:

$$
P(n)=(1-s) P(n-1)+s d+\epsilon(n)
$$

where $s$ determines the influence of the previous preference state, $P(n-1), d$ is the subjective expected payoff and $\epsilon$ is the residual (produced by fluctuations in attention).

In human-robot team interaction this model can be used to model the reliance of the human on the robot team autonomy. The reliance is determined by trust of the human in the autonomy and by selfconfidence of the human in his/her manual control. Therefore, the two alternatives are modeled as the human preference for autonomous or manual control.

Trust in autonomy is the attitude that an agent will help achieve an individual's goals in a situation characterized by uncertainty [65]. Overtrust and undertrust in autonomy can cause overreliance (misuse) and underutilization (disuse), respectively [88]. A review on human trust in autonomy is provided in [65]. Based on (11), trust and self-confidence are estimated as [45]

$$
\begin{array}{r}
T(n)=(1-s) T(n-1)+s d_{c a}(n)+\epsilon(n) \\
S C(n)=(1-s) S C(n-1)+s d_{c m}(n)+\epsilon(n),
\end{array}
$$

where $T$ and $S C$ correspond to trust and selfconfidence, while $d_{c a}$ and $d_{c m}$ are subjective expected payoffs if the task is automated and if it is manual, respectively. The reliance is computed as the preference $P(n)=T(n)-S C(n)$. Therefore, it depends on the dynamical interaction between the trust and the self-confidence.

Another accumulator model, proposed in [107] for the human-robot team interaction, uses a stochastic soft-max choice model that emerges from a driftdiffusion (DD) model. The probability that the the human operator will choose option $A$ is defined as a sigmoidal function:

$$
p_{A}(t+1)=\frac{1}{1+e^{-\mu d(t)}}
$$

where $\mu$ represents the slope of the sigmoidal function. The probability (13) can be represented with a drift-diffusion model:

$$
d z=\alpha d t+\sigma d W, \quad z(0)=0,
$$

where $z$ represents the accumulated evidence in favor of a candidate choice, $\alpha$ is a drift rate representing the signal intensity of the stimulus, and $\sigma W$ is a Wiener process with standard deviation $\sigma$.

The authors of [54] use black-box methods to identify human decision-making behavior in the active role of commanding a swarm. Obtained, linear time-invariant (LTI) system, reveals that the human decision-making process is not passive in the high-frequency range. 


\subsubsection{Constraints on human modeling}

Important constraints that affect the humanrobot team interaction are human workload and situational awareness [18].

The mental workload is the extent to which a task places demands on the human's cognitive resources [100]. The workload increases significantly if the human operator interacts with the individual robots within the team [17]. This corresponds to the interaction on the action layer. Authors of [51] propose that the maximum number of homogeneous and uncoupled robots a single human can manage is determined by the fan out (FO) expression

$$
F O=\frac{N T}{I T}+1
$$

where $N T$ is the neglect time allowed and $I T$ the interaction time required for each robot.

Workload can be reduced by increasing the autonomous capabilities of the robot team, and by establishing the interaction through the subtask layer.

However, with the increase of the robot team autonomy, situational awareness (SA) [36] of the human degrades, reducing human apprehension of the robot team states. It has been shown that if the robot team is involved in the decision-making, the situational awareness is negatively affected [87]. Therefore, the higher the support from the robot team, the greater the risk from complacency, impaired situational awareness and skill degradation. True danger from these effects can occur when the automation fails and the human does not react, has a delayed response or does not have the skill to react properly [86]. Situational awareness can be improved with a suitable interface design.

Important research questions are how can the understanding of the human behavior and constraints aid in the control sharing design and to what extent can the team be included in the decision-making process without inducing negative impact on the human behavior. Furthermore, if the robot team performs multiple subtasks simultaneously, the human can also exhibit multitasking behavior during the interaction. An additional research challenge is to use multitasking decision-making models [91], for the purpose of designing a suitable control architecture for human-robot team interaction.

\section{Interaction aspects for human-robot teams}

In this section we consider possible interaction paradigms between a human and a robotic team in terms of levels of autonomy, allocation of responsibilities and handling multiple subtasks. Furthermore, we provide a review of the interfaces used in this type of interaction.

\subsection{Interaction paradigms}

The approach we take in reviewing types of interaction for human-robot teams is motivated by the degree to which the robot team can perform functions autonomously and by the roles the human and the robot team undertake in the interaction. These roles are majorly influenced by the levels of robot autonomy [53]. The concept of levels of autonomy (LOAs) is introduced in the area of human-machine interaction (HMI):

Definition 3.1. Levels of autonomy are a design aspect that defines which functions should be autonomous and which should be managed by the human [101].

The early research proposes fixed number of discretized levels of autonomy between no autonomy and full autonomy. For example, Sheridan proposes 10 levels of automation in [101], see Table 1 . The concept has been extended to the levels of autonomy for each information-processing system function: information acquisition, information analysis, decision and action selection, and action implementation. For example, high level of autonomy is desirable for information acquisition and information analysis functions, but not for decision making as it causes human skill degradation, complacency and poor situational awareness [89]. Therefore, it is necessary to allow the human to provide commands to the robot team. An important question is to what extent can we include the robot team in the decision-making process.

The concept of levels of autonomy has been considered for human-robot team interaction as well. Each function of the robot team or each robot can have a level of autonomy. In [21] a concept of autonomy spectrum is proposed for human-robot team interaction. An example of the autonomy spectrum is depicted with Figure 4. It is a graphical representation of operating modes (depicted with nodes), with levels of autonomy and functions to be executed along vertical and horizontal graph axes, 


\begin{tabular}{|l|l|}
\hline 1 & The human executes all actions. \\
\hline 2 & $\begin{array}{l}\text { The computer offers complete set of action } \\
\text { alternatives. }\end{array}$ \\
\hline 3 & $\begin{array}{l}\text { The computer offers a selection of action } \\
\text { alternatives. }\end{array}$ \\
\hline 4 & The computer suggests one alternative. \\
\hline 5 & $\begin{array}{l}\text { The computer executes an action au- } \\
\text { tonomously if the human approves. }\end{array}$ \\
\hline 6 & $\begin{array}{l}\text { The computer allows the human a re- } \\
\text { stricted time to veto before automatic ex- } \\
\text { ecution. }\end{array}$ \\
\hline 7 & $\begin{array}{l}\text { The computer executes an action and in- } \\
\text { forms the human. }\end{array}$ \\
\hline 8 & $\begin{array}{l}\text { The computer executes an action and in- } \\
\text { forms the human if asked. }\end{array}$ \\
\hline 9 & $\begin{array}{l}\text { The computer executes an action and in- } \\
\text { forms the human if it decides to. }\end{array}$ \\
\hline 10 & $\begin{array}{l}\text { The computer executes all actions au- } \\
\text { tonomously. }\end{array}$ \\
\hline
\end{tabular}

Table 1: 10 levels of autonomy by Sheridan [101].

respectively. The authors use 10 levels of autonomy, proposed in [101]. The approach allows to determine several operating modes for each function, and to combine them (depicted with lines). This method emphasizes the importance of having multiple operating modes during the task execution. Another important property that needs to be ensured for interaction modes is smooth and seamless transition, termed as sliding scale autonomy [98]. In [70] a sliding autonomy approach is proposed for robot swarms.

In [80] interaction paradigms are introduced for human-robot team interaction through the subtask layer. The authors propose subtask allocation to the human operator and the autonomous controller of the robot team according to the available levels of autonomy. Three interaction paradigms are proposed: direct, complementary and overlapping. Direct interaction paradigm is an interaction in which the human provides commands for all the subtasks. Complementary interaction paradigm is an interaction in which the human provides input to a set of subtasks, while the robot team executes the remaining subtasks autonomously. Overlapping interaction paradigm is an interaction in which the inputs from the human and the robot team autonomy are blended to perform a common subtask.

If we denote the set of all the subtasks that need to be conducted within a mission as $\mathcal{S}$, Table 2 dif-



Figure 4: An example representation of the autonomy spectrum.

ferentiates between the interaction paradigms and provides examples. In many applications, especially the ones that are time-critical, it is necessary to prioritize subtasks. The authors of [87] propose to design an automation matrix which contains weights (representing subtask importance, expected workload and other factors) that are used to prioritize subtasks and determine which of them should be automated. It can be used to allocate responsibility within the interaction and fuse control inputs from the human and the robot team.

However, the priority between subtasks is, so far, determined in advance and cannot dynamically change during the task execution.

\subsection{Interfaces for human-robot team interaction}

Main challenges in the interface design for human-robot interaction are to decide on the suitable command and feedback information and how to represent them appropriately. According to [18], the interface needs to ensure the human understands intentions and behaviors of the robots and the environment. Furthermore, it needs to appropriately allocate human attention to important events and ensure the decision authority of the human. Overall, the interface for human-automation interaction needs to be determined by: purpose (degree to which the automation is used w.r.t. the designer intent), process (if the autonomy level is suitable for a given situation) and performance (reliability, predictability and capability).

\subsubsection{Command interfaces}

In the supervisory role, the human typically interacts with the robot team via a graphical user interface (GUI) (e.g. touch screen [14]). An action of the human supervisor is mapped into high-level commands (e.g. setting goals for the robot team or individual robots, assigning levels of autonomy, 


\begin{tabular}{|c|c|c|c|}
\hline $\begin{array}{c}\text { Interaction } \\
\text { paradigms }\end{array}$ & $\begin{array}{c}\text { Human } \\
\text { responsibilities }\end{array}$ & $\begin{array}{c}\text { Robot team } \\
\text { responsibilities }\end{array}$ & Examples \\
\hline Direct & $\mathcal{S}$ & $\emptyset$ & Teleoperation \\
\hline Complementary & $\mathcal{S}_{h} \subset \mathcal{S}$ & $\mathcal{S}_{a} \subset \mathcal{S}, \mathcal{S}_{h} \cap \mathcal{S}_{a}=\emptyset$ & Semi-autonomy \\
\hline Overlapping & $\mathcal{S}_{h} \subset \mathcal{S}$ & $\mathcal{S}_{a} \subset \mathcal{S}, \mathcal{S}_{h} \cap \mathcal{S}_{a} \neq \emptyset$ & Mixed-initiative \\
\hline
\end{tabular}

Table 2: Properties of the interaction paradigms.

interference in the case of events, etc.). In the active role, the human provides physical commands (e.g. motion and/or force commands), typically via a haptic device, e.g. a master robot in teleoperation scenarios [64].

Human-robot team interaction is asymmetric, as the robot team typically has more degrees of freedom than the human. So far, the challenge of interacting intuitively with highly redundant systems has been tackled for specific application examples only. The research output indicates that the interaction with the robot team through global behaviors in the subtask layer of Figure 2 is suitable, as it reduces the dimensionality of command and feedback information [81]. For example, in order to command a formation behavior, instead of commanding relative distances between individual robots, the human operator can command change of the formation shape using the concept of virtual deformable volumes [2], [29].

From the perspective of control theory, the controllability property of the system can aid in the interface design [80]. Knowing the level of system autonomy implies which states of the robot team should be controllable by the human. In order to ensure controllability of those states, it is necessary to provide sufficient number of command channels. This number conditions the command interface that can be used in the interaction.

\subsubsection{Feedback interfaces}

The feedback signal in human-robot team interaction is typically visual. In the supervisory role, the human receives feedback via GUI and video. In [14] the authors show that if the roles of the human and the robot team are changing during the task execution, the interface should provide dynamical feedback. In [24] authors distinguish between: GUI interfaces for visual representation, warning systems (visual, auditory and haptic) and suggestion systems which propose where the attention should be allocated. The performance in managing multiple UAVs individually proved to be the best with suggestion systems.

In the active role, the human typically receives feedback via a haptic device. Usefulness of the haptic feedback in human-robot team interaction has been confirmed in [83]. Analoguously to the supervisory role, the feedback of continuously changing states should be provided to the human in dynamical form. This conclusion has been made through experimental validation for the control of multiple UAVs in [30] and [31]. In [1] the authors investigate haptic human-robot team interaction with variable formation. The haptic signal informs the human when the swarm is stretched, compressed or reshaped. Relative behavior of the individual robots in the team is a useful feedback information if robots establish multiple contacts with the environment, e.g. in cooperative manipulation tasks. It is shown that wearable haptic devices are a suitable interface in this case [81].

In terms of the appropriate feedback, the human operator should be informed about the states it controls. In that sense, the states which are controllable by the human should also be observable. In order to ensure observability of states, it is necessary to provide sufficient number of feedback channels. This number conditions the feedback interface that can be used in the interaction.

Due to complexity of human-robot team interaction, it is no longer sufficient to provide only the feedback about the system states. It is necessary to represent activity of the automation as well (e.g. the change of the autonomy level) and sensitivity to future activities (e.g. warnings) [115]. The activity of automation of multiple UAVs is examined as a function of interfaces in [106]. The authors show that the interfaces which allow the human to select between different autonomy levels reduce switching costs.

The existing literature shows there are many individual studies on the suitable interface design for specific examples of human-robot team interaction. However, a systematic control theoretical understanding which models the interface as a mapping 
from human action to command signals and from sensor signals to presented information is still missing.

\section{Shared control for human-robot team col- laboration}

Human-robot team interaction represents a collaboration between heterogeneous entities. Therefore, the responsibilities over the task conduction are shared, which is accomplished with shared control approaches. Therefore, we say that the shared control allows to determine the level of collaboration between the human and the robot team. It includes all the control methods between manual and fully autonomous control.

In this section, existing shared control concepts are reviewed. First, we review how the responsibilities among the human and the robot team can be shared. Afterwards, the control theoretical approaches, found in the literature, are summarized. Shared control concepts from a broader area of human-robot interaction are considered if they are deemed as suitable for human-robot team interaction.

\subsection{Collaboration in human-robot team interaction}

Collaboration refers to the interaction in which information, resources and responsabilities are shared among participants. In [21] the authors distinguish between control by behavior (human interacts with each each robot in the team individually) and control by policy (human interacts with the complete robot team). In the context of Figure 2 , control by behavior referes to the control of local behaviors by the human, while control by policy referes to the control of global behaviors by the human. In the control by policy the local behaviors are executed autonomosly. Both control approaches are evaluated in [50]. The authors show that control by policy is more suitable with increasing number of agents. In the case of control by behavior limited interaction intervals can cause inefficient interaction and, in the worst case, failures.

Interaction of the human with individual robots (control by behavior) limits the number of robots within the team. Naturally, it imposes the greatest workload and time-related stress on the human operator [75], [114]. Furthermore, the complexity of the interaction is of order $N, O(N)$. In order to obtain complexity $O(1)$, it is necessary to design autonomous cooperative controller. The reduction of complexity can be achieved with the interaction between the human and a single robot, termed as leader. In this way the human workload is considerably reduced [99]. However, human-robot team interaction is not achieved in this way as the human does not need to understand and act in response to the complete team behavior. Such systems heavily rely on the autonomy of the complete team, which the human typically cannot manage in the case of unpredictable situations.

If the human controls the complete robot team, its team dynamics is managed (control by policy). This approach enables the human to operate on higher levels of abstraction [25]. However, the human is a point of failure of such a system [13]. The solution to this problem is an interaction with a subset of robots in the team [54].

The results in [11] confirm that humans are capable of perceiving a robot team as a single unit. In [13] human collaborates with a robot team at higher levels of abstraction, termed as attractors. They represent states of the overall team dynamics (or its subset). One can also say that attractors are projections of robots' states onto the lower dimensional state space or that they abstract from the individual to the group behavior. They correspond to the introduced concept of global behaviors. We provide a simple and intuitive example of global versus local states.

Example 4.1. The pose of the robot team can represent one of the team overall states. If poses of $N$ robots are $\boldsymbol{x}_{i} \in \mathbb{R}^{2}, i=1, \ldots, N$, the team pose can be their mean value

$$
\boldsymbol{x}=\frac{1}{N} \Sigma_{i=1}^{N} \boldsymbol{x}_{i}
$$

The dimensionality of the overall behavior is reduced from $2 N$ to 2 and the control objective for this team can be $\lim _{t \rightarrow \infty} \boldsymbol{x}(t) \rightarrow \boldsymbol{x}_{h}(t)$, where $\boldsymbol{x}_{h}(t)$ is the trajectory commanded by the human operator.

Authors of [13] additionally impose the stability requirement on the global behaviors. Therefore, the overall team dynamics is stable. This is an important requirement for the shared control design since the human does not need to stabilize the system. Furthermore, the control design on the level of global behaviors is easier in terms of dimensionality [58]. 


\subsection{Shared control approaches}

In this subsection control approaches for humanrobot team interaction are reviewed. Typically, human-robot team interaction is remote and considerable research output treats the problem of shared control design for teleoperation scenarios. We classify the methods into the ones suitable for the complementary interaction paradigm and the ones suitable for overlapping interaction paradigm.

\subsubsection{Shared control for the complementary inter- action paradigm}

Typically, the human is in charge of controlling a global subtask of the overall team behavior. The robot team is in charge of cooperating autonomously [103]. Additionaly, the robots within the team perform a local subtask of collision avoidance, see e.g. [43] and [92]. However, the authors do not consider that the desired commands for different behaviors may be in conflict. This causes unpredictable behaviors of the robot team. Such situations can be resolved by decoupling the dynamics of the overall robot team into the dynamics for the required behaviors. It can be achieved by ensuring the autonomous task is uncontrollable to the human, see e.g. [103] and [80].

Since robot teams are inherently redundant, they can perform multiple subtasks simultaneously. In order to avoid conflicts of control inputs, a nullspace based behavioral control, introduced in Subsection 2.1 can be applied to define, decouple and prioritize multiple subtasks. Using double integrator model of the robots within the team, the authors of [69] assign the responsibility over a set of global behaviors of the team to the human operator. Those are termed as teleoperated tasks and include commanding the position of the robot team mean pose (16) and its variance

$$
\boldsymbol{f}_{v}(\boldsymbol{q})=\frac{1}{N} \Sigma_{i=1}^{N}\left(\boldsymbol{x}_{i}-\boldsymbol{f}_{m}(\boldsymbol{q})\right)^{2},
$$

where $\boldsymbol{q} \in \mathbb{R}^{n N}$ is the stacked vector of generalized coordinates of the robot team.

The subtasks performed by the robot team are dispersion, avoidance of obstacles and the other members of the team. Within the subtask layer, desired control inputs for the low-level controllers within the action layer are computed according to

$$
\dot{\boldsymbol{q}}^{d}=J_{s}^{+} \dot{\boldsymbol{x}}_{s}+\left(I_{n N}-J_{s}^{+} J_{s}\right) \boldsymbol{\psi}_{s},
$$

where $J_{s}$ is the partial derivative of one of the teleoperated subtasks, while $\psi_{s}$ is the sum of partial derivatives of the autonomous subtasks. In this way it is ensured that the teleoperated and autonomous subtasks do not intefere if there are sufficient degrees of freedom. If they interfere, the teleoperated subtasks are of a higher priority over the autonomous subtasks. However, the authors do not prioritize autonomous subtasks. Additionally, the priority is fixed which is not suitable in the case of unpredictable events, nor is it possible to allocate teleoperated tasks to the robot team and vice versa.

The possibility that the robot team interacts and collides with the environment is not treated extensively in the literature. However, cooperative manipulation by the robot team in teleoperation scenarios provides some insights into the appropriate control approaches. In [64] energetic passivity is enforced via passivity-based control. Therefore, the passivity of the system when interacting with a passive environment in guaranteed. Another approach uses impedance control to ensure passivity in interaction with environement, see e.g. [3] and [81].

The reviewed approaches are suitable for the active human role. In the supervisory role, the human typically behaves as a switch. There are also approaches in which the human performs both roles. For example, in [43] the human selects the mode of interaction while the team of UAVs autonomously controls its variable topology. The choice can be made between the global intervention (steering the centroid of the formation to the goal) or the ocal intervention (steering a single UAV). In [54] the human switches manually between two two controllers: the control of the robot team position and the control of the robot team velocity, and provides the input commands to the chosen controller.

The drawback of the reviewed approaches is that the subtask distribution is constant during the task execution and the level of the robot team autonomy is fixed. This is problematic as it reduces the flexibility of the interaction. Furthermore, if multiple subtasks need to be executed, their prioritization is of the fixed order. It would be beneficial to be able to dynamically change the priorities of the subtasks according to the stage of the task execution and to allocate the responsibilities of the subtasks online.

Due to developments of robot autonomy, the performance of the robot team does not necessarely improve with the persistent human command if the robot team knows the goal. However, the human command is suitable in the open-ended missions [18]. Therefore, mixed-initiative control ap- 
proaches that allow the human to be part of the control loop when necessary and to leave the loop when desired, can be suitable in the future.

\subsubsection{Overlapping interaction paradigm}

So far, we reviewed human-robot team interaction in which robot team autonomy complements human capabilities. However, another aspect of shared control can be exhibited in the form of assistance where the human and the robot team jointly perform a common task. Shared control approaches that establish an overlapping interaction paradigm are termed as mixed-initiative. The obtained control commands are a synthesis of the human input and the autonomy input. The block structure of the control loop is depicted with Figure 5.

There is little work done on human-robot team mixed-initiative control. One of the examples is the work of Chipalkatty et al. in [20]. The authors use model predictive control (MPC) to establish a mixed-initiative control of the helicopter robot team, teleoperated by the human operator. The autonomous controller has a built in planner, i.e. the robot team is capable to reach a target autonomously. The human can inject input for the overall robot team behavior and, in this way, interfere with the input from the autonomous controller. The coordination between the robots within the team is handled autonomously. The stability of the global behavior commanded by the human operator is not guaranteed. Instead, it is assumed that the human is capable of stabilizing the corresponding states. Furthermore, the authors disregard the feedback effect of the MPC approach, i.e. the controller predicts the team behavior and the human input on which the human reacts in return. Therefore, stability issues can occur when prediction of the human behavior is autonomous. In this approach it is not necessary to specify levels of autonomy and the MPC approach is suitable only for sufficiently regular human inputs.

Potential mixed-initiative control approaches can be found in the broader area of human-robot interaction. Typically, they are obtained by blending human control inputs and autonomy control inputs. In the remainder we review blending approaches that can be suitable for human-robot team interaction.

Blending mechanisms. Let us denote the human control input as $\boldsymbol{u}_{h}(t)$ and the autonomy control input as $\boldsymbol{u}_{a}(t)$. Their blending is typically linear:

$$
\boldsymbol{u}(t)=K_{h} \boldsymbol{u}_{h}(t)+K_{a} \boldsymbol{u}_{a}(t), \quad K_{h}+K_{a}=I,
$$

where $\boldsymbol{u}(t)$ is the resulting control command, $K_{h}$ and $K_{a}$ are arbitration matrices, and $I$ is an identity matrix. In general, $K_{a}$ quantifies the level of autonomy, and $K_{h}$ the level of collaboration between the human and the autonomy of the robot team.

There are many ways in which arbitration matrices can be selected. The simple approach is to assign fixed and constant values to the matrices [38]. In [112], the author proposes a probabilistic shared control and proves that linear blending is its special case. Furthermore, in this paper shared control is defined as a joint optimization between agreability, safety and efficiency of the interaction. Authors prove that linear blending can generate unsafe sharing with safe human and safe autonomous input. The shared control input is determined as

$$
\begin{aligned}
& \boldsymbol{u}(t)=\boldsymbol{f}^{R *} \\
& \left(\boldsymbol{h}, \boldsymbol{f}^{R}, \boldsymbol{f}\right)^{*}=\arg \max _{\boldsymbol{h}, \boldsymbol{f}^{R}, \boldsymbol{f}} p\left(\boldsymbol{h}, \boldsymbol{f}^{R}, \boldsymbol{f} \mid \boldsymbol{z}_{1: t}^{h}, \boldsymbol{z}_{1: t}^{R}, \boldsymbol{z}_{1: t}^{f}\right)
\end{aligned}
$$

where $\boldsymbol{h}, \boldsymbol{f}^{R}, \boldsymbol{f}$ are human, robot, and dynamic obstacle trajectories, and $\boldsymbol{z}_{1: t}^{h}, \boldsymbol{z}_{1: t}^{R}, \boldsymbol{z}_{1: t}^{f}$ their corresponding measurements. The probabilistic shared control is determined as

$$
p\left(\boldsymbol{h}, \boldsymbol{f}^{R}, \boldsymbol{f} \mid \boldsymbol{z}_{1: t}\right)=\psi\left(\boldsymbol{h}, \boldsymbol{f}^{R}\right) p\left(\boldsymbol{h} \mid \boldsymbol{z}_{1: t}^{h}\right) p\left(\boldsymbol{f}^{R}, \boldsymbol{f} \mid \overline{\boldsymbol{z}}_{1: t}\right)
$$

where $\psi\left(\boldsymbol{h}, \boldsymbol{f}^{R}\right)=\exp \left(-\frac{1}{2 \gamma}\left(\boldsymbol{h}-\boldsymbol{f}^{R}\right)\left(\boldsymbol{h}-\boldsymbol{f}^{R}\right)^{T}\right)$ is the interaction function between the human and the robot with the coupling factor $\gamma$. Dynamical prediction function of the human behavior is $p\left(\boldsymbol{h} \mid \boldsymbol{z}_{1: t}^{h}\right)$. The dynamical prediction of the autonomy is $p\left(\boldsymbol{f}^{R}, \boldsymbol{f} \mid \overline{\boldsymbol{z}}_{1: t}\right)$.

Arbitration of the human input and the robot team input can be achieved using game-theoretical approaches, see e.g. [68]. Recently, the arbitration based on the estimation of human trust and selfconfidence has been validated. In [96] a mixedinitiative bilateral teleoperation is proposed for the control of a single mobile robot. It uses trust models to scale the manual and the autonomous control inputs with a human-to-robot trust and to scale feedback with a robot-to-human trust. A passivity-based controller successfully manages the time-varying scales and communication time delays.

The arbitration can also be applied on the parameters of the low-level controller of the action layer. 


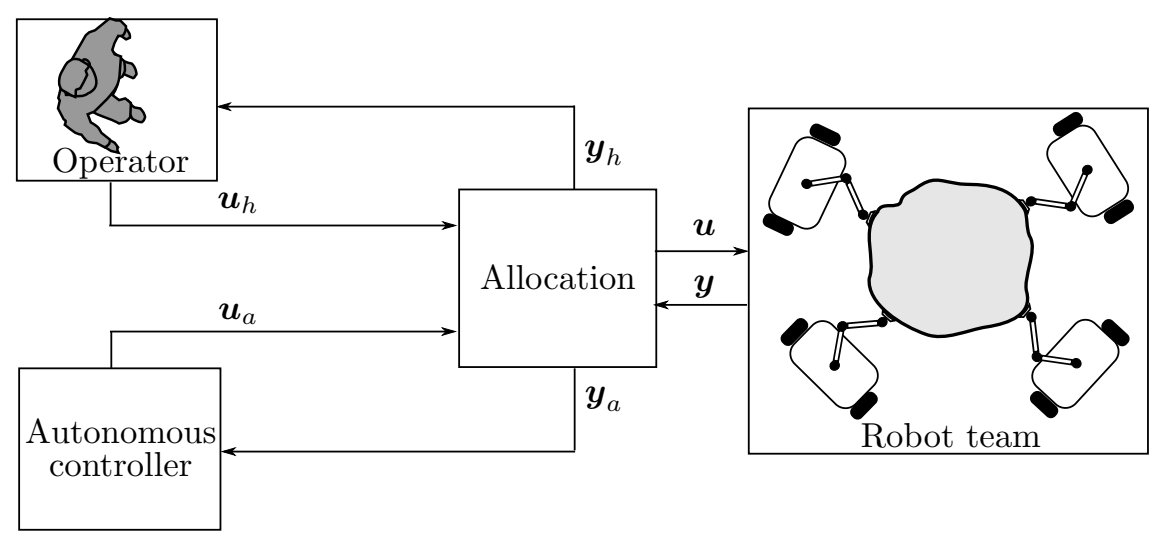

Figure 5: Block scheme of the control loop for the human-robot team interaction in a cooperative manipulation task.

For example, in order to obtain safe and intuitive assistance, an approach to the allocation of control authority is achieved using a human-inspired decision-making model (13). The authors of [48] treat the problem of shared control of a mobile assistive robot (MAR) by solving simultaneously 3 low level sub-tasks: follow a path, avoid collisions and mitigate human fatigue. For each subtask a drift-diffusion decision-making model is used for gain scheduling of the low-level control parameters of admittance or impedance controller:

$$
c(t)=p_{A}(t+1) \underline{c}+\left(1-p_{A}(t+1)\right) \bar{c}
$$

where $\underline{c}$ and $\bar{c}$ represent the upper and lower bounds of the control parameter $c$. However, the authors disregard the problem of subtask conflicts, since they do not consider their interference in experimental evaluation. A similar approach is used in [22] for a single task and bilateral teleoperation scenario. Experimental results show that the decision-making models have potential for intuitive mixed-initiative interaction.

In the mixed initiave shared control, the human modeling is important, since it is necessary to determine the most appropriate autonomous control input based on the human behavior to accomplish a satisfactory assistance. In [32] the authors propose the linear arbitration with constant selection matrices, and with the autonomous control input that is based on the prediction of the human intent. This approach adapts to the robot confidence in itself, to the user confidence and the user type. It uses machine learning for the estimation.

We can conclude that tasks should be decomposed into multiple subtasks represented by stable global and local behaviors. Furthermore, these sub-



Figure 6: Block structure of the general hierarchical shared control architecture for human-robot team interaction. Based on a desired goal of the interaction and the environment state subtasks are generated and prioritized. Allocation of subtasks to the human and the robot team is dynamical and determined depending on the available levels of autonomy, current self-confidence of the human and its trust in automation. Low-level controllers receive desired control inputs either from human or from the built-in robot team planners.

tasks should be prioritized according to the current state of the environment and allocated dynamically to the human and/or the autonomous controller of the robot team as depicted in Figure 6. Dynamical distribution of subtasks among the human and the robot team is termed as trading control [55], [59]. As far as the authors know, this has not been done so far for human-robot team interaction. 


\section{Conclusion}

Control sharing in human-robot team interaction is summarized in this article through the effects that each component of the closed-loop, given with Figure 1, has on the overall performance. Therefore, we reviewed human, robot team, interface and control aspects of the interaction. Following conclusions can be made based on the reviewed literature:

- With the increase of autonomous capabilities of robots, role of humans in the interaction is not reduced. On the contrary, human gains more high-level responsibilities. Therefore, it is important to include human in the control loop as a decision-making dynamical system.

- Autonomous capabilities of robot teams are described by the autonomy spectrum, which includes levels of autonomy assigned to each subtask that the robot team can perform. Combinations of levels of autonomy define interaction paradigms between the human and the robot team. Paradigms represent the design aspects of the interaction and indicate shared control requirements.

- The human should interact with the robot team on the subtask level by managing its global behaviors. In this way, the high dimensional robot team state-space is projected onto the lower-dimensional space of the global behavior for which the control design is easier.

- Interface in human-robot team interaction should provide intuitive mappings to resolve the inherent asymmetry.

- Mixed-initiative shared control approaches enable both human and robot team to make decisions and can benefit from the human behavior modeling.

For a better overview, reviewed literature is sorted in Table 3 with respect to the elements of the shared control loop and the taxonomy for the human-robot team interaction.

\section{Future work}

The review of the available literature indicates the need to perceive the human as a team member. There are a number of research challenges within the area of human-robot team interaction; we highlight some of them:
- Models of the human cognitive process are necessary within the control theoretical context. Therefore, decision-making dynamical models from cognitive psychology might provide a useful construct to tackle the problem of heterogeneity.

- Developing a control architecture which tunes the assistance of the robot team based on the monitored workload and situational awareness can enable human and robots to function as a team. Therefore, mixed-initiative shared control is a promising control concept for further research.

- Another important aspect is how to effectively and appropriately choose suitable level of autonomy. A lot of potential lies in approaches that optimize level of autonomy with respect to the human confidence in performing certain task, i.e. by modeling trust in automation and human self-confidence.

- A major challenge is to design appropriate oneto-many mappings between the human and the robot team from the control theoretical perspective in order to be able to formally analyze interaction properties during the task execution.

- Robot teams as redundant systems can perform multiple subtasks simultaneously. A major challenge is to design, prioritize and distribute the subtasks among the human and the robot team autonomy dynamically. This largely depends on the state of the environment and the available levels of autonomy.

- If multiple subtasks are considered, the control loop needs to handle multitasking situations from the human and control perspective. Therefore, incorporating multitasking decision-making models into control loop would be a challenging research goal.

Overall, sophisticated shared control strategies that rely on mixed-initiative interaction, multitasking capabilities, dynamical prioritization and distribution of subtasks should bring us closer to a peer-to-peer human-robot team interaction.

\section{References}

[1] C. K. Alder, S. J. McDonald, M. B. Colton, M. A. Goodrich, Toward haptic-based management of small 


\begin{tabular}{ll}
\hline Topic & References \\
\hline \hline Supervisory role and human-on-the-loop & {$[26],[45],[17],[91],[95],[75]$} \\
\hline Active role and human-in-the-loop & {$[64],[73],[43],[104][54],[96],[105],[62],[18]$,} \\
& {$[69],[71],[92],[103],[60],[61],[42],[22]$} \\
\hline Human modeling (Subsection 2.2) & {$[17],[51][36],[65],[24],[26],[44],[45],[93]$,} \\
& {$[107],[86],[91],[54],[15],[14],[97],[110]$} \\
\hline \multirow{2}{*}{ Robot team control (Subsection 2.1) } & {$[102],[79],[47],[108],[27],[56],[76],[34],[77]$,} \\
\hline \multirow{2}{*}{ Robot team surveys (Subsection 2.1) } & {$[66],[109],[85]$} \\
\hline Interaction paradigms (Subsection 3.1) & {$[79],[39],[33],[16],[47],[5]$} \\
& {$[101],[86],[89],[95],[6],[28],[8],[21],[113]$,} \\
\hline Interfaces (Subsection 3.2) & {$[80],[106][115][41]$} \\
\hline Adjustable control (Section 4) & {$[17],[51],[14],[29],[1],[2],[31],[30],[52],[83]$,} \\
\hline Mixed-initiative control (Section 4) & $[43],[99],[106],[82],[9]]$ \\
\hline \hline
\end{tabular}

Table 3: An overview of surveyed literature for control sharing in human-robot team interaction.

swarms in cordon and patrol, in: Swarm/Human Blended Intelligence Workshop (SHBI), 2015, IEEE, $1-8,2015$.

[2] J. Alonso-Mora, S. H. Lohaus, P. Leemann, R. Siegwart, P. Beardsley, Gesture based human - Multi-robot swarm interaction and its application to an interactive display, in: 2015 IEEE International Conference on Robotics and Automation (ICRA), ISSN 1050-4729, 5948-5953, doi:10.1109/ICRA.2015.7140033, 2015.

[3] M. Angerer, S. Music, S. Hirche, Port-Hamiltonian model based control for human-robot team interaction, in: Proceedings of 2017 IEEE International Conference on Robotics and Automation (ICRA), 2017.

[4] G. Antonelli, F. Arrichiello, S. Chiaverini, The null-space-based behavioral control for autonomous robotic systems, Intelligent Service Robotics 1 (1) (2008) 27-39, ISSN 18612784, doi:10.1007/s11370-007-0002-3, URL http://dx.doi.org/10.1007/s11370-007-0002-3.

[5] T. Arai, E. Pagello, L. E. Parker, Editorial: Advances in multi-robot systems, IEEE Transactions on robotics and automation 18 (5) (2002) 655-661.

[6] M. Baker, H. A. Yanco, Autonomy mode suggestions for improving human-robot interaction., in: SMC (3), 2948-2953, 2004.

[7] T. Balch, R. C. Arkin, Behavior-based formation control for multirobot teams, IEEE Transactions on robotics and automation 14 (6) (1998) 926-939.

[8] J. Beer, A. D. Fisk, W. A. Rogers, Toward a framework for levels of robot autonomy in human-robot interaction, Journal of Human-Robot Interaction 3 (2) (2014) 74.

[9] H. Boessenkool, D. A. Abbink, C. J. Heemskerk, F. C. van der Helm, J. G. Wildenbeest, A task-specific analysis of the benefit of haptic shared control during telemanipulation, IEEE Transactions on Haptics 6 (1) (2013) 2-12.

[10] J. Brookshire, S. Singh, R. Simmons, Preliminary results in sliding autonomy for assembly by coor- dinated teams, in: Intelligent Robots and Systems, 2004.(IROS 2004). Proceedings. 2004 IEEE/RSJ International Conference on, vol. 1, IEEE, 706-711, 2004.

[11] D. S. Brown, M. A. Goodrich, S.-Y. Jung, S. C. Kerman, Two Invariants of Human Swarm Interaction, Journal of Human-Robot Interaction 5 (1) (2015) 131.

[12] D. S. Brown, S. Y. Jung, M. A. Goodrich, Balancing human and inter-agent influences for shared control of bio-inspired collectives, in: 2014 IEEE International Conference on Systems, Man, and $\mathrm{Cy}$ bernetics (SMC), ISSN 1062-922X, 4123-4128, doi: 10.1109/SMC.2014.6974582, 2014.

[13] D. S. Brown, S. C. Kerman, M. A. Goodrich, Humanswarm Interactions Based on Managing Attractors, in: Proceedings of the 2014 ACM/IEEE International Conference on Human-robot Interaction, HRI '14, ACM, New York, NY, USA, ISBN 978-1-45032658-2, 90-97, doi:10.1145/2559636.2559661, URL http://doi.acm.org/10.1145/2559636.2559661, 2014.

[14] D. J. Bruemmer, D. A. Few, R. L. Boring, J. L. Marble, M. C. Walton, C. W. Nielsen, Shared understanding for collaborative control, IEEE Transactions on Systems, Man, and Cybernetics-Part A: Systems and Humans 35 (4) (2005) 494-504.

[15] M. Cao, A. Stewart, N. E. Leonard, Integrating human and robot decision-making dynamics with feedback: Models and convergence analysis, in: Decision and Control, 2008. CDC 2008. 47th IEEE Conference on, IEEE, 1127-1132, 2008.

[16] Y. U. Cao, A. S. Fukunaga, A. Kahng, Cooperative mobile robotics: Antecedents and directions, Autonomous robots 4 (1) (1997) 7-27.

[17] J. Y. Chen, M. J. Barnes, M. Harper-Sciarini, Supervisory control of multiple robots: Human-performance issues and user-interface design, IEEE Transactions on Systems, Man, and Cybernetics, Part C (Applications 
and Reviews) 41 (4) (2011) 435-454.

[18] J. Y. C. Chen, M. J. Barnes, Human Agent Teaming for Multirobot Control: A Review of Human Factors Issues, IEEE Transactions on Human-Machine Systems 44 (1) (2014) 13-29.

[19] M. Chiou, R. Stolkin, G. Bieksaite, N. Hawes, K. L. Shapiro, T. S. Harrison, Experimental analysis of a variable autonomy framework for controlling a remotely operating mobile robot, in: Intelligent Robots and Systems (IROS), 2016 IEEE/RSJ International Conference on, IEEE, 3581-3588, 2016

[20] R. Chipalkatty, G. Droge, M. B. Egerstedt, Less is more: Mixed-initiative model-predictive control with human inputs, IEEE Transactions on Robotics 29 (3) (2013) 695-703.

[21] G. Coppin, F. Legras, Autonomy spectrum and performance perception issues in swarm supervisory control, Proceedings of the IEEE 100 (3) (2012) 590-603.

[22] J. Corredor, J. Sofrony, A. Peer, Decision-making model for adaptive impedance control of teleoperation systems, IEEE Transactions on Haptics

[23] J. Cortes, S. Martinez, T. Karatas, F. Bullo, Coverage control for mobile sensing networks, IEEE Transactions on robotics and Automation 20 (2) (2004) 243255.

[24] J. W. Crandall, M. L. Cummings, M. Della Penna, P. M. de Jong, Computing the effects of operator attention allocation in human control of multiple robots, IEEE Transactions on Systems, Man, and Cybernetics-Part A: Systems and Humans 41 (3) (2011) 385-397.

[25] J. W. Crandall, M. A. Goodrich, D. R. Olsen, C. W. Nielsen, Validating human-robot interaction schemes in multitasking environments, IEEE Transactions on Systems, Man, and Cybernetics - Part A: Systems and Humans 35 (4) (2005) 438-449, ISSN 1083-4427, doi: 10.1109/TSMCA.2005.850587.

[26] M. L. Cummings, J. P. How, A. Whitten, O. Toupet, The impact of human-automation collaboration in decentralized multiple unmanned vehicle control, Proceedings of the IEEE 100 (3) (2012) 660-671.

[27] J. P. Desai, J. P. Ostrowski, V. Kumar, Modeling and control of formations of nonholonomic mobile robots, IEEE transactions on Robotics and Automation 17 (6) (2001) 905-908.

[28] M. Desai, H. A. Yanco, Blending human and robot inputs for sliding scale autonomy, in: ROMAN 2005. IEEE International Workshop on Robot and Human Interactive Communication, 2005., IEEE, 537-542, 2005.

[29] M. Diana, J. P. de la Croix, M. Egerstedt, Deformablemedium affordances for interacting with multi-robot systems, in: 2013 IEEE/RSJ International Conference on Intelligent Robots and Systems, ISSN 2153-0858, 5252-5257, doi:10.1109/IROS.2013.6697116, 2013.

[30] B. Donmez, M. L. Cummings, H. D. Graham, Auditory decision aiding in supervisory control of multiple unmanned aerial vehicles, Human factors 51 (5) (2009) $718-729$.

[31] B. Donmez, H. Graham, M. Cummings, Assessing the impact of haptic peripheral displays for UAV operators, Tech. Rep., DTIC Document, 2008.

[32] A. D. Dragan, S. S. Srinivasa, A policy-blending formalism for shared control, The International Journal of Robotics Research 32 (7) (2013) 790-805.
[33] G. Dudek, M. Jenkin, E. Milios, A taxonomy of multirobot systems, Robot teams: From diversity to polymorphism (2002) 3-22.

[34] W. B. Dunbar, R. M. Murray, Distributed receding horizon control for multi-vehicle formation stabilization, Automatica 42 (4) (2006) 549-558.

[35] M. Egerstedt, X. Hu, Formation constrained multiagent control, IEEE transactions on robotics and automation 17 (6) (2001) 947-951.

[36] M. R. Endsley, Toward a theory of situation awareness in dynamic systems, Human Factors: The Journal of the Human Factors and Ergonomics Society 37 (1) (1995) 32-64.

[37] S. Erhart, S. Hirche, Internal force analysis and load distribution for cooperative multi-robot manipulation, IEEE Transactions on Robotics 31 (5) (2015) 12381243 .

[38] P. Evrard, A. Kheddar, Homotopy-based controller for physical human-robot interaction, in: RO-MAN 2009The 18th IEEE International Symposium on Robot and Human Interactive Communication, IEEE, 1-6, 2009.

[39] A. Farinelli, L. Iocchi, D. Nardi, Multirobot systems: a classification focused on coordination, IEEE Transactions on Systems, Man, and Cybernetics, Part B (Cybernetics) 34 (5) (2004) 2015-2028, ISSN 1083-4419, doi:10.1109/TSMCB.2004.832155.

[40] J. A. Fax, R. M. Murray, Information flow and cooperative control of vehicle formations, IEEE transactions on automatic control 49 (9) (2004) 1465-1476.

[41] D. Feth, B. A. Tran, R. Groten, A. Peer, M. Buss, Shared-control paradigms in multi-operator-singlerobot teleoperation, in: Human Centered Robot Systems, Springer, 53-62, 2009.

[42] A. Franchi, P. R. Giordano, C. Secchi, H. I. Son, H. H. Bülthoff, A passivity-based decentralized approach for the bilateral teleoperation of a group of UAVs with switching topology, in: Robotics and Automation (ICRA), 2011 IEEE International Conference on, IEEE, 898-905, 2011.

[43] A. Franchi, C. Secchi, M. Ryll, H. H. Bulthoff, P. R. Giordano, Shared control: Balancing autonomy and human assistance with a group of quadrotor UAVs, IEEE Robotics \& Automation Magazine 19 (3) (2012) $57-68$.

[44] F. Gao, A. S. Clare, J. C. Macbeth, M. Cummings, Modeling the impact of operator trust on performance in multiple robot control, AAAI, 2013.

[45] J. Gao, J. D. Lee, Extending the decision field theory to model operators' reliance on automation in supervisory control situations, IEEE Transactions on Systems, Man, and Cybernetics-Part A: Systems and Humans 36 (5) (2006) 943-959.

[46] V. Gazi, Swarm aggregations using artificial potentials and sliding-mode control, IEEE Transactions on Robotics 21 (6) (2005) 1208-1214, ISSN 1552-3098, doi:10.1109/TRO.2005.853487.

[47] V. Gazi, B. Fidan, Coordination and Control of Multiagent Dynamic Systems: Models and Approaches, Springer Berlin Heidelberg, Berlin, Heidelberg, ISBN 978-3-540-71541-2, 71-102, 2007.

[48] M. Geravand, C. Werner, K. Hauer, A. Peer, An Integrated Decision Making Approach for Adaptive Shared Control of Mobility Assistance Robots, International Journal of Social Robotics (2016) 1-18. 
[49] B. P. Gerkey, M. J. Matarić, A formal analysis and taxonomy of task allocation in multi-robot systems, The International Journal of Robotics Research 23 (9) (2004) 939-954.

[50] M. A. Goodrich, T. W. McLain, J. D. Anderson, J. Sun, J. W. Crandall, Managing Autonomy in Robot Teams: Observations from Four Experiments, in: Proceedings of the ACM/IEEE International Conference on Human-robot Interaction, HRI '07, ACM, New York, NY, USA, ISBN 978-1-59593617-2, 25-32, doi:10.1145/1228716.1228721, URL http://doi.acm.org/10.1145/1228716.1228721, 2007.

[51] M. A. Goodrich, M. Quigley, K. Cosenzo, Task Switching and Multi-Robot Teams, Springer Netherlands, Dordrecht, ISBN 978-1-4020-3389-6, 185-195, 2005.

[52] W. B. Griffin, W. R. Provancher, M. R. Cutkosky, Feedback strategies for telemanipulation with shared control of object handling forces, Presence: Teleoperators and Virtual Environments 14 (6) (2005) 720-731.

[53] B. Hardin, M. A. Goodrich, On Using Mixedinitiative Control: A Perspective for Managing Large-scale Robotic Teams, in: Proceedings of the 4th ACM/IEEE International Conference on Human Robot Interaction, HRI '09, ACM, New York, NY, USA, ISBN 978-1-60558-4041, 165-172, doi:10.1145/1514095.1514126, URL http://doi.acm.org/10.1145/1514095.1514126, 2009.

[54] T. Hatanaka, N. Chopra, M. Fujita, Passivity-based bilateral human-swarm-interactions for cooperative robotic networks and human passivity analysis, in: 2015 54th IEEE Conference on Decision and Control (CDC), IEEE, 1033-1039, 2015.

[55] S. Hayati, S. Venkataraman, Design and implementation of a robot control system with traded and shared control capability, in: Robotics and Automation, 1989. Proceedings., 1989 IEEE International Conference on, IEEE, 1310-1315, 1989.

[56] Y. Jin, A. A. Minai, M. M. Polycarpou, Cooperative real-time search and task allocation in UAV teams, in: Decision and Control, 2003. Proceedings. 42nd IEEE Conference on, vol. 1, IEEE, 7-12, 2003.

[57] H. Kitano, S. Tadokoro, I. Noda, H. Matsubara, T. Takahashi, A. Shinjou, S. Shimada, RoboCup Rescue: search and rescue in large-scale disasters as a domain for autonomous agents research, in: Systems, Man, and Cybernetics, 1999. IEEE SMC '99 Conference Proceedings. 1999 IEEE International Conference on, vol. 6, ISSN 1062-922X, 739-743 vol.6, doi: 10.1109/ICSMC.1999.816643, 1999.

[58] A. Kolling, P. Walker, N. Chakraborty, K. Sycara, M. Lewis, Human Interaction With Robot Swarms: A Survey, IEEE Transactions on Human-Machine Systems 46 (1) (2016) 9-26.

[59] D. Kortenkamp, R. P. Bonasso, D. Ryan, D. Schreckenghost, Traded control with autonomous robots as mixed initiative interaction, in: AAAI Symposium on Mixed Initiative Interaction, 89-94, 1997.

[60] D. Lee, Semi-autonomous teleoperation of multiple wheeled mobile robots over the internet, in: ASME 2008 Dynamic Systems and Control Conference, American Society of Mechanical Engineers, 147154, 2008.

[61] D. Lee, Passive decomposition and control of non- holonomic mechanical systems, IEEE Transactions on Robotics 26 (6) (2010) 978-992.

[62] D. Lee, A. Franchi, H. I. Son, C. Ha, H. H. Bülthoff, P. R. Giordano, Semiautonomous haptic teleoperation control architecture of multiple unmanned aerial vehicles, IEEE/ASME Transactions on Mechatronics 18 (4) (2013) 1334-1345.

[63] D. Lee, M. W. Spong, Bilateral teleoperation of multiple cooperative robots over delayed communication networks: application, in: Proceedings of the 2005 IEEE International Conference on Robotics and $\mathrm{Au}-$ tomation, IEEE, 360-365, 2005.

[64] D. Lee, M. W. Spong, Bilateral teleoperation of multiple cooperative robots over delayed communication networks: theory, in: Proceedings of the 2005 IEEE International Conference on Robotics and Automation, IEEE, 360-365, 2005.

[65] J. D. Lee, K. A. See, Trust in automation: Designing for appropriate reliance, Human Factors: The Journal of the Human Factors and Ergonomics Society 46 (1) (2004) 50-80.

[66] N. E. Leonard, E. Fiorelli, Virtual leaders, artificial potentials and coordinated control of groups, in: Decision and Control, 2001. Proceedings of the 40th IEEE Conference on, vol. 3, IEEE, 2968-2973, 2001.

[67] M. A. Lewis, K.-H. Tan, High precision formation control of mobile robots using virtual structures, Autonomous robots 4 (4) (1997) 387-403.

[68] Y. Li, K. P. Tee, W. L. Chan, R. Yan, Y. Chua, D. K. Limbu, Role adaptation of human and robot in collaborative tasks, in: Robotics and Automation (ICRA), 2015 IEEE International Conference on, IEEE, 56025607, 2015.

[69] C.-W. Lin, M.-H. Khong, Y.-C. Liu, Experiments on Human-in-the-Loop Coordination for Multirobot System With Task Abstraction, IEEE Transactions on Automation Science and Engineering 12 (3) (2015) 981-989.

[70] L. Lin, M. A. Goodrich, Sliding Autonomy for UAV Path-Planning: Adding New Dimensions to Autonomy Management, in: Proceedings of the 2015 International Conference on Autonomous Agents and Multiagent Systems, AAMAS '15, International Foundation for Autonomous Agents and Multiagent Systems, Richland, SC, ISBN 978-1-4503-3413-6, 16151624, 2015.

[71] Y.-C. Liu, Task-space coordination control of bilateral human-swarm systems, Journal of the Franklin Institute 352 (1) (2015) 311-331.

[72] S. G. Loizou, V. Kumar, Mixed initiative control of autonomous vehicles, in: Robotics and Automation, 2007 IEEE International Conference on, IEEE, 14311436, 2007.

[73] P. Malysz, S. Sirouspour, Trilateral teleoperation control of kinematically redundant robotic manipulators, The International Journal of Robotics Research 30 (13) (2011) 1643-1664.

[74] J. L. Marble, D. J. Bruemmer, D. A. Few, Lessons learned from usability tests with a collaborative cognitive workspace for human-robot teams, in: Systems, Man and Cybernetics, 2003. IEEE International Conference on, vol. 1, IEEE, 448-453, 2003.

[75] S. Mau, J. Dolan, Scheduling for humans in multirobot supervisory control, in: 2007 IEEE/RSJ International Conference on Intelligent Robots 
and Systems, ISSN 2153-0858, 1637-1643, doi: 10.1109/IROS.2007.4399340, 2007.

[76] P. McDowell, J. Chen, B. Bourgeois, UUV teams, control from a biological perspective, in: OCEANS'02 MTS/IEEE, vol. 1, IEEE, 331-337, 2002.

[77] N. Michael, M. M. Zavlanos, V. Kumar, G. J. Pappas, Distributed multi-robot task assignment and formation control, in: Robotics and Automation, 2008. ICRA 2008. IEEE International Conference on, IEEE, 128-133, 2008

[78] C. A. Miller, H. Funk, R. Goldman, J. Meisner, P. Wu, Implications of adaptive vs. adaptable UIs on decision making: Why automated adaptiveness is not always the right answer, in: Proceedings of the 1st international conference on augmented cognition, 22-27, 2005.

[79] R. M. Murray, Recent research in cooperative control of multivehicle systems, Journal of Dynamic Systems, Measurement, and Control 129 (5) (2007) 571-583.

[80] S. Musić, S. Hirche, Classification of human-robot team interaction paradigms, in: 1st IFAC Conference on Cyber-Physical \& Human-Systems, 2016, 2016.

[81] S. Musi, G. Salvietti, P. Bude gen. Dohmann, F. Chinello, D. Prattichizzo, S. Hirche, Human-MultiRobot Teleoperation for Cooperative Manipulation Tasks using Wearable Haptic Devices, in: IEEE/RSJ International Conference on Intelligent Robots and Systems, accepted, 2017.

[82] Y. Nevatia, T. Stoyanov, R. Rathnam, M. Pfingsthorn, S. Markov, R. Ambrus, A. Birk, Augmented autonomy: Improving human-robot team performance in Urban search and rescue, in: 2008 IEEE/RSJ International Conference on Intelligent Robots and Systems, ISSN 2153-0858, 2103-2108, doi: 10.1109/IROS.2008.4651034, 2008.

[83] S. Nunnally, P. Walker, M. Lewis, N. Chakraborty, K. Sycara, Using haptic feedback in human robotic swarms interaction, in: Proceedings of the Human Factors and Ergonomics Society Annual Meeting, vol. 57, SAGE Publications Sage CA: Los Angeles, CA, 1047-1051, 2013.

[84] R. Olfati-Saber, Flocking for multi-agent dynamic systems: Algorithms and theory, IEEE Transactions on automatic control 51 (3) (2006) 401-420.

[85] R. Olfati-Saber, R. M. Murray, Consensus problems in networks of agents with switching topology and timedelays, IEEE Transactions on automatic control 49 (9) (2004) 1520-1533.

[86] L. Onnasch, C. D. Wickens, H. Li, D. Manzey, Human performance consequences of stages and levels of automation: An integrated meta-analysis, Human Factors 56 (3) (2014) 476-488.

[87] R. Parasuraman, M. Barnes, K. Cosenzo, S. Mulgund, Adaptive automation for human-robot teaming in future command and control systems, Tech. Rep., DTIC Document, 2007.

[88] R. Parasuraman, V. Riley, Humans and automation: Use, misuse, disuse, abuse, Human Factors: The Journal of the Human Factors and Ergonomics Society 39 (2) (1997) 230-253.

[89] R. Parasuraman, T. B. Sheridan, C. D. Wickens, A model for types and levels of human interaction with automation, IEEE Transactions on Systems, Man, and Cybernetics - Part A: Systems and Humans 30 (3) (2000) 286-297, ISSN 1083-4427.
[90] L. E. Parker, Multiple mobile robot systems, in: Springer Handbook of Robotics, Springer, 921-941, 2008.

[91] J. R. Peters, V. Srivastava, G. S. Taylor, A. Surana, M. P. Eckstein, F. Bullo, Human supervisory control of robotic teams: integrating cognitive modeling with engineering design, IEEE Control Systems 35 (6) (2015) 57-80.

[92] E. J. Rodríguez-Seda, J. J. Troy, C. A. Erignac, P. Murray, D. M. Stipanovic, M. W. Spong, Bilateral teleoperation of multiple mobile agents: Coordinated motion and collision avoidance, IEEE Transactions on Control Systems Technology 18 (4) (2010) 984-992.

[93] R. M. Roe, J. R. Busemeyer, J. T. Townsend, Multialternative decision field theory: A dynamic connectionst model of decision making., Psychological review 108 (2) (2001) 370.

[94] E. M. Roth, M. L. Hanson, C. Hopkins, V. Mancuso, G. L. Zacharias, Human in the loop evaluation of a mixed-initiative system for planning and control of multiple UAV teams, in: Proceedings of the $\mathrm{Hu}-$ man Factors and Ergonomics Society Annual Meeting, vol. 48, SAGE Publications Sage CA: Los Angeles, CA, 280-284, 2004.

[95] H. A. Ruff, S. Narayanan, M. H. Draper, Human interaction with levels of automation and decision-aid fidelity in the supervisory control of multiple simulated unmanned air vehicles, Presence: Teleoperators and virtual environments 11 (4) (2002) 335-351.

[96] H. Saeidi, F. McLane, B. Sadrfaidpour, E. Sand, S. Fu, J. Rodriguez, J. Wagner, Y. Wang, Trust-based mixedinitiative teleoperation of mobile robots, in: American Control Conference (ACC), 2016, IEEE, 6177-6182, 2016.

[97] D. Schuster, S. Ososky, F. Jentsch, E. Phillips, C. Lebiere, W. A. Evans, A research approach to shared mental models and situation assessment in future robot teams, in: Proceedings of the Human Factors and Ergonomics Society Annual Meeting, vol. 55, SAGE Publications Sage CA: Los Angeles, CA, 456460, 2011.

[98] B. Sellner, F. W. Heger, L. M. Hiatt, R. Simmons, S. Singh, Coordinated Multiagent Teams and Sliding Autonomy for Large-Scale Assembly, Proceedings of the IEEE 94 (7) (2006) 1425-1444, ISSN 0018-9219, doi:10.1109/JPROC.2006.876966.

[99] T. Setter, H. Kawashima, M. Egerstedt, Team-level properties for haptic human-swarm interactions, in: 2015 American Control Conference (ACC), ISSN 07431619, 453-458, doi:10.1109/ACC.2015.7170777, 2015.

[100] T. Sheridan, H. Stassen, Definitions, models and measures of human workload, in: Mental Workload, Springer, 219-233, 1979.

[101] T. B. Sheridan, W. L. Verplank, Human and computer control of undersea teleoperators, Tech. Rep., DTIC Document, 1978.

[102] D. H. Shim, H. J. Kim, S. Sastry, Decentralized nonlinear model predictive control of multiple flying robots, in: 42nd IEEE International Conference on Decision and Control (IEEE Cat. No.03CH37475), vol. 4, ISSN 0191-2216, 3621-3626 vol.4, doi:10.1109/CDC.2003.1271710, 2003.

[103] D. Sieber, S. Musić, S. Hirche, Multi-robot manipulation controlled by a human with haptic feedback, in: Intelligent Robots and Systems (IROS), 2015 
IEEE/RSJ International Conference on, IEEE, 2440$2446,2015$.

[104] S. Sirouspour, Modeling and control of cooperative teleoperation systems, IEEE Transactions on Robotics 21 (6) (2005) 1220-1225.

[105] H. I. Son, A. Franchi, L. L. Chuang, J. Kim, H. H. Bulthoff, P. R. Giordano, Human-centered design and evaluation of haptic cueing for teleoperation of multiple mobile robots, IEEE transactions on cybernetics 43 (2) (2013) 597-609.

[106] P. Squire, G. Trafton, R. Parasuraman, Human control of multiple unmanned vehicles: effects of interface type on execution and task switching times, in: Proceedings of the 1st ACM SIGCHI/SIGART conference on Human-robot interaction, ACM, 26-32, 2006.

[107] A. Stewart, M. Cao, A. Nedic, D. Tomlin, N. Leonard, Towards human-robot teams: Model-based analysis of human decision making in two-alternative choice tasks with social feedback, Proceedings of the IEEE 100 (3) (2012) 751-775.

[108] T. G. Sugar, V. Kumar, Control of cooperating mobile manipulators, IEEE Transactions on robotics and automation 18 (1) (2002) 94-103.

[109] D. Swaroop, J. Hedrick, String stability of interconnected systems, IEEE transactions on automatic control 41 (3) (1996) 349-357.

[110] K. Sycara, C. Lebiere, Y. Pei, D. Morrison, M. Lewis, Abstraction of analytical models from cognitive models of human control of robotic swarms, in: International Conference on Cognitive Modeling, University of Pittsburgh, 2015.

[111] H. G. Tanner, A. Jadbabaie, G. J. Pappas, Stable flocking of mobile agents, part I: fixed topology, in: 42nd IEEE International Conference on Decision and Control (IEEE Cat. No.03CH37475), vol. 2, ISSN 0191-2216, 2010-2015 Vol.2, doi: 10.1109/CDC.2003.1272910, 2003.

[112] P. Trautman, Assistive planning in complex, dynamic environments: a probabilistic approach, in: Systems, Man, and Cybernetics (SMC), 2015 IEEE International Conference on, IEEE, 3072-3078, 2015.

[113] P. Walker, S. Nunnally, M. Lewis, N. Chakraborty, K. Sycara, Levels of automation for human influence of robot swarms, in: Proceedings of the Human Factors and Ergonomics Society Annual Meeting, vol. 57, SAGE Publications Sage CA: Los Angeles, CA, 429433, 2013.

[114] J. Wang, M. Lewis, Human control for cooperating robot teams, in: 2007 2nd ACM/IEEE International Conference on Human-Robot Interaction (HRI), ISSN 2167-2121, 9-16, 2007.

[115] D. D. Woods, J. Tittle, M. Feil, A. Roesler, Envisioning human-robot coordination in future operations, IEEE Transactions on Systems, Man, and Cybernetics, Part C (Applications and Reviews) 34 (2) (2004) 210-218.

[116] H. A. Yanco, J. L. Drury, A taxonomy for human-robot interaction, in: Proceedings of the AAAI Fall Symposium on Human-Robot Interaction, 111-119, 2002.

[117] D. Zhang, G. Xie, J. Yu, L. Wang, Adaptive task assignment for multiple mobile robots via swarm intelligence approach, Robotics and Autonomous Systems 55 (7) (2007) 572-588. 